\section{Healing with hyaluronan}

Specialized $\mathrm{T}$ cells in the skin lay a sugary foundation for macrophage migration into wounds, according to a study on page 1269. Jameson and colleagues show that the absence of dendritic epidermal $\gamma \delta \mathrm{T}$ cells (DETCs) removes the impetus for skin cells to secrete hyaluronan, an extracellular glycan that is required for macrophage entry into wounds. Without macrophages, wounds can't heal.

Wound healing is initiated when DETCs recognize an unknown antigen on damaged skin cells. Neutrophils and, later, macrophages migrate to the wound site; both cell types are needed for complete healing. This group had previously shown that wound repair breaks down in the absence of DETCs due to a lack of keratinocyte growth factors FGF-7 and FGF-10, which are produced by DETCs in wounds and stimulate keratinocyte regeneration.

Jameson et al. noted that nonhealing wounds in DETC-deficient mice were less inflamed than

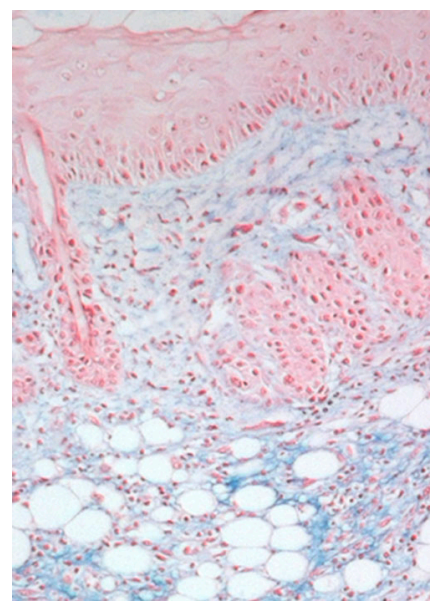

Efficient macrophage migration into wounded skin requires hyaluronan (blue). normal wounds. They now show that, though neutrophils arrived at the wound on schedule, macrophages showed up late. The macrophage tardiness could be traced back to the lack of FGF-7. In normal wounds, FGF-7 was found to trigger the production of hyaluronan by neighboring keratinocytes and hyaluronan was needed to recruit macrophages. If hyaluronan (or FGF-7) was added back to the wounds, the macrophages returned and healing was restored.

Impaired wound healing is prevalent in patients with diabetes and rheumatoid arthritis. The authors hope to investigate whether impaired DETC function may be to blame in these human diseases. JEM

\section{Neutrophil-DC close encounters}

On page 1281, van Gisbergen and colleagues describe for the first time a direct interaction between neutrophils and dendritic cells (DCs). The event triggers DC activation, primes T helper 1 (Th1) cells, and may help explain why Th1 responses to some pathogens are impaired in the absence of neutrophils.

Neutrophils have been shown to influence the priming of Th1 responses indirectly: they secrete cytokines and chemokines that promote Th1 responses and recruit DCs into infected tissues. But van Gisbergen et al. now show that neutrophils don't always keep their distance. They can interact with DCs directly through binding of the C-type lectin DC-SIGN on DCs to specific carbohydrate structures on the neutrophil integrin Mac-1.

Neutrophil-DC binding triggered activation of the DCs as measured by DC up-regulation of CD86 and secretion of interleukin-12. But this only happened if the neutrophils were first activated, suggesting that DC activation only occurs in an inflammatory setting. Neutrophilactivated DCs went on to prime interferon- $\gamma$-producing Th1 cells.

One possible consequence of this interaction, the authors speculate, might be transfer of antigens from the neutrophils to the DCs, although this idea remains to be tested. Another open question is whether neutrophils receive signals as part of the relationship with DCs. JEM
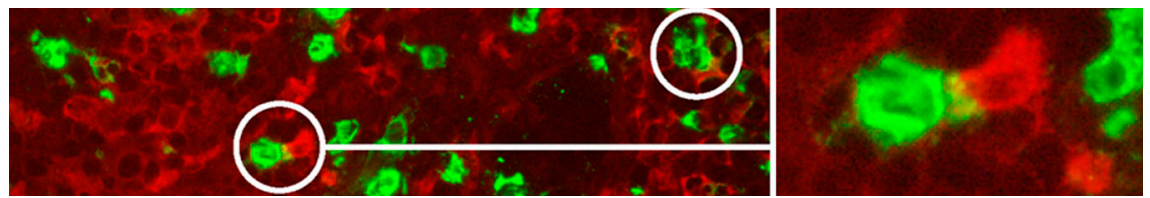

The dendritic cell lectin DC-SIGN (red) binds to carbohydrate structures on the neutrophil integrin Mac-1 (green).

\section{Polymerase pinch hitters}

On page 1191, Delbos and colleagues provide the first proof that the errorprone polymerase $\eta$ (pol $\eta$ ) is responsible for mutations at A-T base pairs during somatic hypermutation (SHM) of immunoglobulin $(\mathrm{lg})$ genes in mice. But when pol $\eta$ is removed from mice, another sloppy enzyme, not previously thought to contribute to SHM, can fill in as a pinch hitter.

SHM generates high affinity antibodies in response to antigenic challenge; it does so by introducing point mutations into the antigenbinding regions of $\mathrm{B}$ cell antibody genes. Mutations at $\mathrm{C}-\mathrm{G}$ base pairs during SHM are the work of the enzyme AID (activation-induced cytidine deaminase), which turns cytosine into uracil. A-T mutations have been harder to explain. Errorprone polymerases are thought be the culprits behind A-T mutation, but specific roles for these enzymes have been difficult to assign, as mice lacking individual polymerases have thus far shown no defects in SHM.

Pol $\eta$ has been the primary suspect charged with mutating A-T base pairs, as the pattern of errors made by pol $\eta$ in vitro is reminiscent of that seen in mutated Ig loci. In addition, humans lacking pol $\eta$ have fewer A-T mutations in their Ig genes than normal. Delbos et al. now solidify the evidence by showing that elimination of pol $\eta$ in mice decreases Ig gene mutations at A-T base pairs.

The few A-T mutations that occurred in the absence of poln-to the authors' surprise-bore the signature of another polymerase, polк, which does not normally meddle in SHM. The authors suggest that the mismatch repair protein complex MSH2-MSH6, recently shown to recruit poln to AID-induced $\mathrm{U}-\mathrm{G}$ mismatches, may in poln's absence instead bind polк. The polк would then cause mutations at nearby A-T sites. JEM 